$551.511 .6,551.551 .5$

\title{
A Corrected Turbulon Model Theory of Turbulent Diffusion and Its Application
}

\author{
By H. Matsuoka \\ Meteorological Institute, Kyoto University \\ (Manuscript received 21 March 1960)
}

\begin{abstract}
Inoue's turbulon theory is modified by taking into account the age of turbulons and his functional form for Lagrangian correlation function is corrected to coincide with Grant's result.

A turbulon theory of the relative diffusion of moving origin type is constructed by introducing a plausible assumption that the turbulons can be effectively separated into two groups viz. that of smaller turbulons contributing only to the diffusion and that of larger turbulons contributing only to the displacement of the center of cluster, and the same results as those predicted by Batchelor's similarity theory are obtained.
\end{abstract}

\section{Introduction}

The determination of the functional form of the so-called Lagrangian correlation function $R(\xi)$ is of primary importance for the study of turbulent diffusion of intermediate dispersion time, and is attempted by many investigators from various points of view.

Among the recent studies, two of E. Inoue's (1950b) and A. M. Grant's (1957) drew the author's attention. Inoue's theory is built on the assumption that the disintegrating turbulons of any rank conserve their proper life-time; while Grant's theory is proceeded by correcting the ordinary mixing-length theory. The discrepancy of the results is found by the anthor's discrimination of the both theories and is eliminated successfully by the correction of Inoue's theory. Applying the reasonable results thus obtained, some basic problems of turbulent diffusion for intermediate dispersion time are studied in this paper.

\section{Lagrangian correlation function $\boldsymbol{R}(\xi)$}

Following Inoue's, velocity component $v(t)$ for any one materialized point at any time $t$ along $y$-direction, perpendicular to $x$-axis oriented to the direction of steady uniform mean flow $(U)$, within statistically isotropic, homogeneous and stationary turbulent field, can be represented by

$$
v(t)=v_{\infty}(t)+\cdots \cdots+v_{n}(t)+\cdots \cdots+v_{0}(t),
$$

where $v_{n}(t)$ is the $y$-component of the proper velocity of turbulon of rank $n$ which surrounds the specified materialized point, and the velocity component at an interval of $\xi$ later is given by

$$
\begin{gathered}
v(t+\xi)=v_{\infty}(t+\xi)+\cdots \cdots+v_{n}(t+\xi)+\cdots \cdots \\
+v_{0}(t+\xi) .
\end{gathered}
$$

By the presumption of the individuality of every rank of turbulon, the Lagrangian correlation function $R(\xi)$ defined by

$$
R(\xi)=\frac{\overline{v(t) v(t+\xi)}}{\overline{v^{2}}}
$$

can be represented as follows :

$$
\begin{aligned}
R(\xi)=R_{\infty}(\xi) \frac{\bar{v}_{\infty}^{2}}{\bar{v}^{2}}+\cdots \cdots & +R_{n}(\xi) \frac{\bar{v}_{n}^{2}}{\bar{v}^{2}}+\cdots \cdots \\
& +R_{0}(\xi) \frac{\bar{v}^{2} 0}{\bar{v}^{2}} .
\end{aligned}
$$

The presumption of the conservation of the original proper velocity of the disintegrating turbulons during the proper life-time $\tau_{n}$ led Inoue to conclude that

$$
R_{n}(\xi)= \begin{cases}1 & \text { for } \xi \leqq \tau_{n} \\ 0 & \text { for } \xi \geqq \tau_{n}\end{cases}
$$

and with a plausible assumption for the spectral function of kinetic energy of the turbulons

$$
F(\tau)= \begin{cases}0 & \text { for } \tau<\tau_{\infty} \\ 1 /\left(\tau_{0}-\tau_{\infty}\right) & \text { for } \tau_{\infty} \leqq \tau \leqq \tau_{0} \\ 0 & \text { for } \tau>\tau_{0}\end{cases}
$$


he derived

$$
R(\xi)=\left\{\begin{array}{cc}
1 & \text { for } \xi \leq \tau_{\infty} \\
1-\left(\xi-\tau_{\infty}\right) /\left(\tau_{0}-\tau_{\infty}\right) & \text { or approximately } \\
1-\left(\xi / \tau_{0}\right) & \text { for } \tau_{\infty} \leqq \xi \leqq \tau_{0} \\
0 & \text { for } \xi \geqq \tau_{0} .
\end{array}\right.
$$

His supposition (4), the foundation of his study, is discussed critically by the author. He supposed that the material point considered at time $t$ was surrounded only by the fresh turbulons having full life-time, but there is no reason why all of them are fresh. In fact, some of them may be old, and have no full original life-time. Taking into account the idea above mentioned, Inoue's result is corrected as follws.

In derivation of $R_{n}(\xi)$, we must classify the turbulons of rank $n$ surrounding the point considered at $t$ into two groups by the remaining effective life-time " $\mathrm{e}$ " or age of the turbulons represented by $\tau_{n}-e$. For the turbulons of $e>\xi$ we have $R(\xi)=1$, while for the turbulons of $e \leqq \xi$ we have $R_{n}(\xi)=0$, and the ratio of the number of the tunbulons belonging to the first group $e>\xi$, to that of the second group $e \leqq \xi$ is considered as $1-\xi / \tau_{n}$ : $\xi / \tau_{n}$ by a reasonable assumption of equally appearance of the chance for the turbulons of different ages. Thus we eventually have

$$
R_{n}(\xi)= \begin{cases}1-\xi / \tau_{n} & \text { for } 0 \leqq \xi<\tau_{n} \\ 0 & \text { for } \xi \geqq \tau_{n} .\end{cases}
$$

Then, specifying rank $n$ so as to be $\tau_{n+1}<$ $\xi \leqq \tau_{n}$ for given $\xi$, we have

$$
\begin{aligned}
R(\xi)=\frac{\bar{v}^{2}}{\bar{v}^{2}}\left(1-\frac{\xi}{\tau_{n}}\right) & +\frac{\bar{v}^{2}{ }_{n-1}}{\bar{v}^{2}}\left(1-\frac{\xi}{\tau_{n-1}}\right)+\cdots \cdots \\
& +\frac{\bar{v}^{2}{ }_{0}}{\bar{v}^{2}}\left(1-\frac{\xi}{\tau_{0}}\right)
\end{aligned}
$$

Assuming the continuous distribution of turbulons between $\left(0, \tau_{0}\right)$, we have from (8)

$$
R(\xi)=\int_{0}^{\tau} F(\tau) R_{\tau}(\xi) d \tau
$$

where

Table 1.

\begin{tabular}{c|c|c|c|c|c|c|c|c|c|c|c}
\hline$\xi / \tau_{0}$ & 0 & 0.1 & 0.2 & 0.3 & 0.4 & 0.5 & 0.6 & 0.7 & 0.8 & 0.9 & 1.0 \\
\hline $1-\frac{\xi}{\tau_{0}}+\frac{\xi}{\tau_{0}} \log \frac{\xi}{\tau_{0}}$ & 1 & 0.670 & 0.478 & 0.338 & 0.234 & 0.154 & 0.094 & 0.051 & 0.021 & 0.005 & 0.000 \\
\hline $\exp \left(-4 \xi / \tau_{0}\right)$ & 1 & 0.670 & 0.449 & 0.301 & 0.202 & 0.135 & 0.091 & 0.061 & 0.041 & 0.027 & 0.018 \\
\hline
\end{tabular}

$$
R_{\tau}(\xi)= \begin{cases}1-\xi / \tau & \text { for } 0 \leqq \xi \leqq \tau \\ 0 & \text { for } \xi \geqq \tau\end{cases}
$$

and

$$
F(\tau)= \begin{cases}1 / \tau_{0} & \text { for } \tau \leqq \tau_{0} \\ 0 & \text { for } \tau>\tau_{0}\end{cases}
$$

Then we have

$$
R(\xi)= \begin{cases}1-\frac{\xi}{\tau_{0}}+\frac{\xi}{\tau_{0}} \log \frac{\xi}{\tau_{0}} & \left(0 \leqq \xi \leqq \tau_{0}\right) \\ 0 & \left(\xi>\tau_{0}\right) .\end{cases}
$$

Since the functional from of $R(\xi)$ thus obtained is in perfect agreement with that derived already by A.M. Grant in a different way in 1957, we can say that correction of Inoue's theory is successfully done. It is added to notice that our expression of $R(\xi)$ shows a fair numerical agreement with a familiar expression $R(\xi)=\exp (-\xi / A)$ if we take $A=\tau_{0} / 4$ giving the same value of $\mathrm{La}^{-}$ grangian scale of turbulence or $\int_{0}^{\infty} R(\xi) d \xi$ for both expressions (see Table 1).
The eddy diffusivity $K(\xi)$ and $\bar{y}^{2}(\xi)$, the expected or mean square distance traveled (in the $y$-direction) by any particle of the fluid in the time $\xi$, which are expressed by the well-known Taylor's (1922) formulae

and

$$
\begin{aligned}
& K(\xi) \equiv \frac{1 d \bar{y}^{2}}{2}=\bar{v}^{2} \int_{0}^{\xi} R\left(\xi^{\prime}\right) d \xi^{\prime} \\
& \overline{y^{2}(\xi)}=2 \bar{v}^{2} \int_{0}^{\xi} \int_{0}^{\xi^{\prime}} R\left(\xi^{\prime \prime}\right) d \xi^{\prime \prime} d \xi^{\prime}
\end{aligned}
$$

can be evaluated, by applying the correlation function $R(\xi)$ expressed by (12). And the results are as follows:

$K(\xi)= \begin{cases}\bar{v}^{2} \xi\left(1-\frac{3}{4} \frac{\xi}{\tau_{0}}+\frac{\xi}{2 \tau_{0}} \log \frac{\xi}{\tau_{0}}\right) & \left(\xi \leqq \tau_{0}\right) \\ \frac{\bar{v}^{2} \tau_{0}}{4} & \left(\xi \geqq \tau_{0}\right)\end{cases}$

and

$\bar{y}^{2}(\xi)= \begin{cases}\bar{v}^{2} \xi^{2}\left(1-\frac{11}{18} \frac{\xi}{\tau_{0}}+\frac{\xi}{3 \tau_{0}} \log \frac{\xi}{\tau_{0}}\right) & \left(\xi \leqq \tau_{0}\right) \\ \bar{v}^{2} \tau_{0}\left(\frac{\xi}{2}-\frac{\tau_{0}}{9}\right) & \left(\xi \geqq \tau_{0}\right)\end{cases}$ 


\section{Two-dimensional diffusion of a cluster re- leased instantaneously at a point source}

Study of diffusion of moving origin type such as smoke puffs has been in focus in the field of the study of atmospheric diffusion since Batchelor's $(1950,1952)$ theory of relative diffusion was presented. He noticed that the relative diffusion is clearly controlled by the small-scale component of turbulence so long as the parcels are fairly close together and he derived some useful formulae predicted by similarity principles. The basic idea, originally proposed by L. F. Richardson (1926), that only the comparatively small scale turbulence components contribute mainly to the eddy diffusion process while the comparatively large scale turbulence components contribute mainly to the displacement of the cluster as a whole or of the center of the cluster mass when the growing cluster has intermediate size, is introduced in a simplifyed form by Inoue (1951a, 1951b, 1952), and by treating the horizontal diffusion of fixed source type he get the similar result to Batchelor's.

A similar presumption to Inoue's is taken in the present study. Referring to the possibility of the labeling of the size of turbulons by their life-time $(\tau$ 's $)$ and that of the size of growing cluster by dispersion time $(\xi)$, the author assumes that the turbulons with $\tau<k \xi$ effectively contribute only to the diffusion of cluster particles and the turbulons with $\tau \geqq k \xi$ effectively contribute only to the translaion of cluster as a whole, where $k$ is a certain constant to be $0<k<1$. It must be noticed that the above-mentioned assumption has no physical reality, but it may be valid in conventional sense, and no serious error may be expected as far as we discuss the resultant statistical nature.

If we denote

$y_{i}(\xi): y$-coordinate of each particle of cluster at any dispersion time $\xi$ relative to the fixed coordinate system whose origin is the original source point, designating any one particle by suffix $i$,

$y_{r_{i}}(\xi): y$-coordinate of any one particle designated by suffix $i$ at that instant relative to the center of cluster,

$y_{g}(\xi): y$-coordinate of the center of the cluster at that time relative to the fixed coordinate system whose origin is the origiral source point, we have

$$
y_{i}(\xi)=y_{g}(\xi)+y_{r_{i}}(\xi)
$$

and, employing the commonly used blacket notation to represent the average values of the whole particles composing the cluster i. e. $[Q]=\frac{1}{n} \sum_{i=1}^{n} Q_{i}$, where $n$ is the total number of particles composing the cluster, we have

$$
\left[y^{2}(\xi)\right]=y^{2}{ }_{g}(\xi)+\left[y^{2}{ }_{r}(\xi)\right]
$$

by inserting the definition of the center of cluster or $\left[y_{r}\right]=0$.

The above equation (17) is valid for a cluster of single puff. Repeating the release of such puff in sufficient large number so as to be capable of estimating the mean value of each term of equation (17) to make a single puff a sample, we have

$$
\overline{\left[y^{2}\right]}=\overline{y^{2}{ }_{g}}+\overline{\left[y_{r}^{2}\right]} \text {, }
$$

where bar notation means the mean value of all samples. It is clear that $\left[\overline{\left.y^{2}(\xi)\right]}\right.$ is identical with $\bar{y}^{2}$ which is the mean square distance traveled by the trial particle at $\xi$ from the fixed origin and identical with $\bar{y}^{2}$ represented by equation (14) in the previous section. And, by the assumed condition of uniformity of the turbulence, we know that each $\bar{y}_{r}^{2}$ taken at any moving origin gives the same value independent of the location of moving origin itself. Accordingly we have

$$
\left[\overline{y^{2}}\right]=\overline{y^{2}} .
$$

Consequently we have from (18)

$$
\bar{y}^{2}=\overline{y_{g}^{2}}+\overline{y_{r}^{2}}
$$

and

$$
\frac{1}{2} \frac{d \bar{y}^{2}}{d \xi}=\frac{1 d \overline{y_{g}}}{2} d \xi=\frac{1 d \overline{y_{r}}}{2}
$$

or

$$
K(\xi)=K_{g}(\xi)+K_{r}(\xi) \text {. }
$$

The evaluation of each term of equation (21) can be carried out as follows.

(i) $K(\xi)$ is already given by (15) i. e.

$K(\xi)= \begin{cases}\bar{v}^{2} \xi\left(1-\frac{3}{4} \frac{\xi}{\tau_{0}}+\frac{\xi}{2 \tau_{0}} \log \frac{\xi}{\tau_{0}}\right) & \left(\xi \leqq \tau_{0}\right) \\ \frac{\bar{v}^{2} \tau_{0}}{4} & \left(\xi \geqq \tau_{0}\right)\end{cases}$ 
(ii) By our assumption the effective turbulons for $K_{r}$ at $\xi$ are limited by $\tau \leqq k \xi$. Accordingly we have by (11) and (9) when $k \xi \leqq \tau_{0}$

$$
K_{r}(\xi)=\bar{v}^{\prime 2} \int_{0}^{\xi} \int_{0}^{k \xi} \frac{1}{k \xi} R_{\tau}\left(\xi^{\prime}\right) d \tau d \xi^{\prime},
$$

where $\bar{v}^{\prime 2}$ is the mean total kinetic energy of effective turbulons for $K_{r}$ and by the same assumption as (11) $\bar{v}^{\prime 2}=\left(k \xi / \tau_{0}\right) \bar{v}^{2}$. The evaluation of (23) can be done by putting $\bar{v}^{\prime 2}$ and $k \xi$ in place of $\bar{v}^{2}$ and $\tau_{0}$ in (22) respectively and taking the case of $\xi \geqq \tau_{0}$ in (22) since $\xi>k \xi$, we have

$$
K_{r}(\xi)=\frac{\bar{v}^{\prime 2} k \xi}{4}=\frac{\bar{v}^{2} k^{2} \xi^{2}}{4 \tau_{0}} .
$$

While, when $k \xi \geqq \tau_{0}$, it is easily seen that

$$
K_{r}(\xi)=K(\xi)=\frac{\bar{v}^{2} \tau_{0}}{4} .
$$

(iii) By the aid of the relation (21) i. e. $K_{g}=K-K_{r}$, the expression for $K_{g}(\xi)$ is obtained as follows:

$$
K_{g}(\xi)= \begin{cases}\bar{v}^{2} \xi\left(1-\frac{3+k^{2}}{4} \frac{\xi}{\tau_{0}}+\frac{\xi}{2 \tau_{0}} \log \frac{\xi}{\tau_{0}}\right) & \left(\xi \leqq \tau_{0}\right) \\ \frac{\bar{v}^{2}}{4 \tau_{0}}\left(\tau^{2}{ }_{0}-k^{2} \xi^{2}\right) & \left(\tau_{0} \leqq \xi\right. \\ 0 & \left.\leqq \tau_{0} / k\right) \\ 0 & \left(\xi \geqq \tau_{0} / k\right) .\end{cases}
$$

Next the evaluation of each term of equation (20) can be carried out as follows:

$\overline{y^{2}(\xi)}$ is given by (16), and integrating (24), (25) and (26), we have

$$
\overline{y^{2}{ }_{r}(\xi)}= \begin{cases}\frac{\bar{v}^{2} k^{2} \xi^{3}}{6 \tau_{0}} & \left(\xi \leqq \tau_{0} / k\right) \\ \frac{\bar{v}^{2} \tau^{2}{ }_{0}}{6}\left(3 \frac{\xi}{\tau_{0}}-\frac{2}{k}\right) & \left(\xi \geqq \tau_{0} / k\right)\end{cases}
$$

and

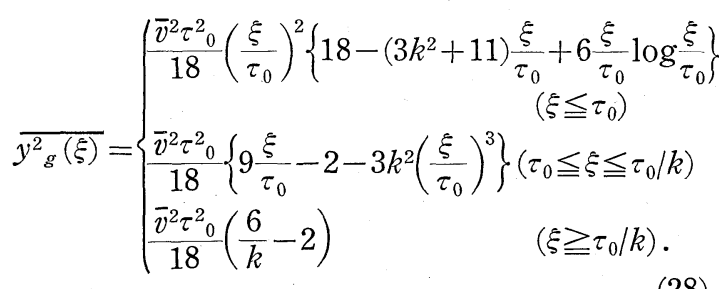

A graphical illustration of the above results is shown by Figs. 1 and 2, where $k$ is taken to be 0.5 as an example.

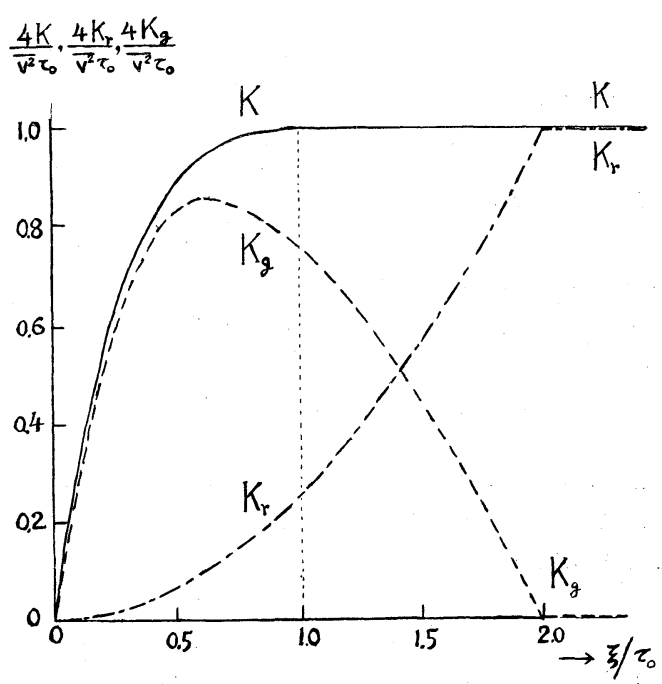

Fig. 1. [Graphical illustration of $K(\xi), K_{r}(\xi)$ and $K_{g}(\xi)$.

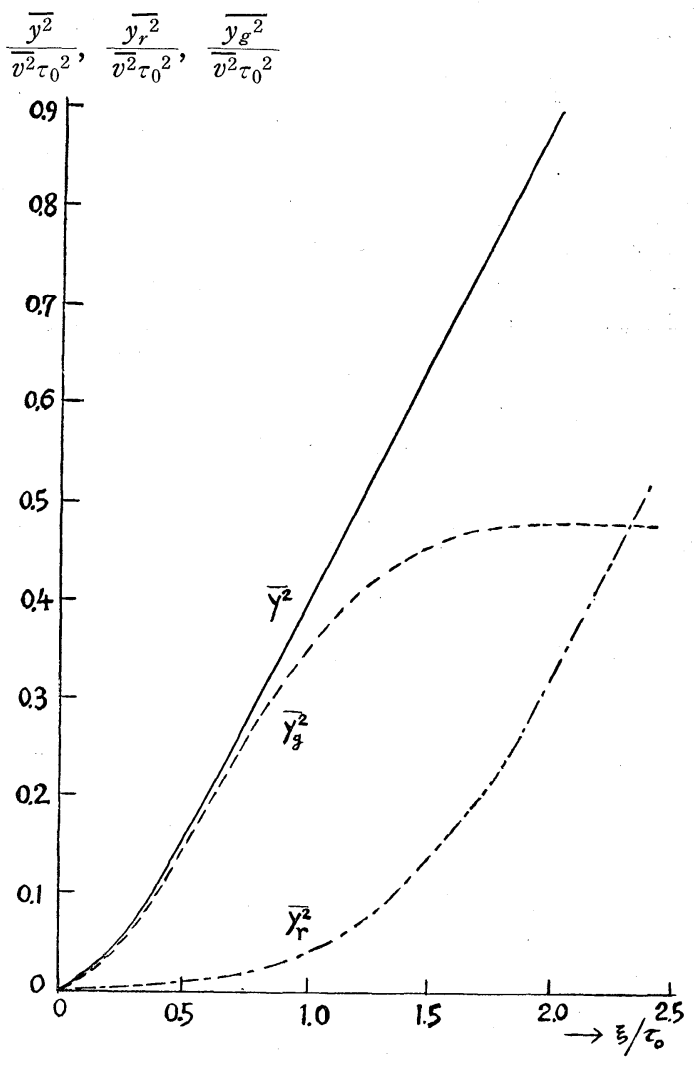

Fig. 2. Graphical illustration of $\bar{y}^{2}(\xi), \bar{y}^{2}{ }_{r}(\xi)$ and $\bar{y}^{2} g(\xi)$. 
4. Note on relative diffusion coefficient during the intermediate dispersion time, $\boldsymbol{K}_{r}(\xi)$

Allowing to regard $\sqrt{\bar{y}^{2}}$ or $l$ as a representative length scale of the diffusion phenomenon, L. F. Richardson's (1926) law of $K \propto l^{4 / 3}$ can be deduced from our result; i. e. from (24) and (27), eliminating $\xi$ we have

$$
K_{r}(\xi)=\frac{1}{4}\left(36 \frac{k^{2} \bar{v}^{2}}{\tau_{0}}\right)^{\frac{1}{3}}\left(\sqrt{\overline{y_{r}^{2}}}\right)^{4 / 3} .
$$

The same results with ours were already given by Batchelor $(1950,1952)$, i. e. Batchelor's results

$$
\frac{d \overline{y^{2}}}{d \xi}=c \varepsilon \xi^{2} \text { and } \overline{y^{2}}=\frac{c}{3} \varepsilon \xi^{3}
$$

are respectively identical with our expressions of (24) and (27) if we put

$$
c \varepsilon=\frac{\bar{v}^{2} k^{2}}{2 \tau_{0}} \text {. }
$$

Rough order of $\frac{\bar{v}^{2} k^{2}}{2 \tau_{0}}$ or $c \varepsilon$ can be known by Gifford's (1957) graphs showing the experimental data of smoke puffs. From his graphs, we have

$\overline{y^{2}} \approx \approx 10 \xi^{3}$ for Frenkiel and Katzs' (1956) experiments near the ground i.e. $5 \sim 8 \mathrm{~m}$ height above the ground,

$\overline{y^{2}} \approx 0.4 \xi^{3}$ for Kellogg's (1956) experiments in the stratosphere and referring to Charnock's (1951) analysis we may infer

$\overline{y^{2}{ }_{r}} \approx 1.5 \xi^{3}$ for levels of $1 \sim 2 \mathrm{~km}$ above the ground. Accordingly we can estimate that $\bar{v}^{2} k^{2} / 6 \tau_{0}$ or $c \varepsilon / 3$ is 10 (C.G.S.) near the ground, 100 at the height of $1 \sim 2 \mathrm{~km}$ above the ground and $10^{-1}$ in the stratosphere level.

\section{Remarks}

(i) Inoue (1951b) identifies his result of $R(\xi)=1-\left(\xi / \tau_{0}\right)$ by a well-known method of dimensional analysis on the basis of similarity hypotheses. It seems to be a serious objection against our corrected result of $R(\xi)=1-\left(\xi / \tau_{0}\right)+\left(\xi / \tau_{0}\right) \log \left(\xi / \tau_{0}\right), \quad$ However the establishment of the similarity hypothses may be doubtful at least for the present problem ; in fact, Inoue gives no satisfactory illustration for its establishment. Therefore the objection may be removed.

(ii) By the quite similar reason as that of the correction for Inoue's (1950b) expression of $R_{n}(\xi)$, Lagrangian correlation function for turbulon of rank $n, R_{n}(t)$ and $f_{n}(r)$, basic functions for deriving Eulerian correlation function $R(t)$ and Taylor-Karmanian correlation functions $f(r)$ and $g(r)$, (Inoue 1950a) must be corrected as follows :

$R_{n}(t)=\left\{\begin{array}{ll}1 & \left(t \leqq T_{n}\right) \\ 0 & \left(t \geqq T_{n}\right)\end{array}\right.$ into $R_{n}(t)= \begin{cases}1-t / T_{n}\left(t \leqq T_{n}\right) \\ 0 & \left(t \geqq T_{n}\right)\end{cases}$ and

$f_{n}(r)=\left\{\begin{array}{ll}1 & \left(r \leqq \Lambda_{n}\right) \\ 0 & \left(r \geqq \Lambda_{n}\right)\end{array}\right.$ into $f_{n}(r)= \begin{cases}1-r / A_{n} & \left(r \leqq \Lambda_{n}\right) \\ 0 & \left(r \geqq \Lambda_{n}\right),\end{cases}$

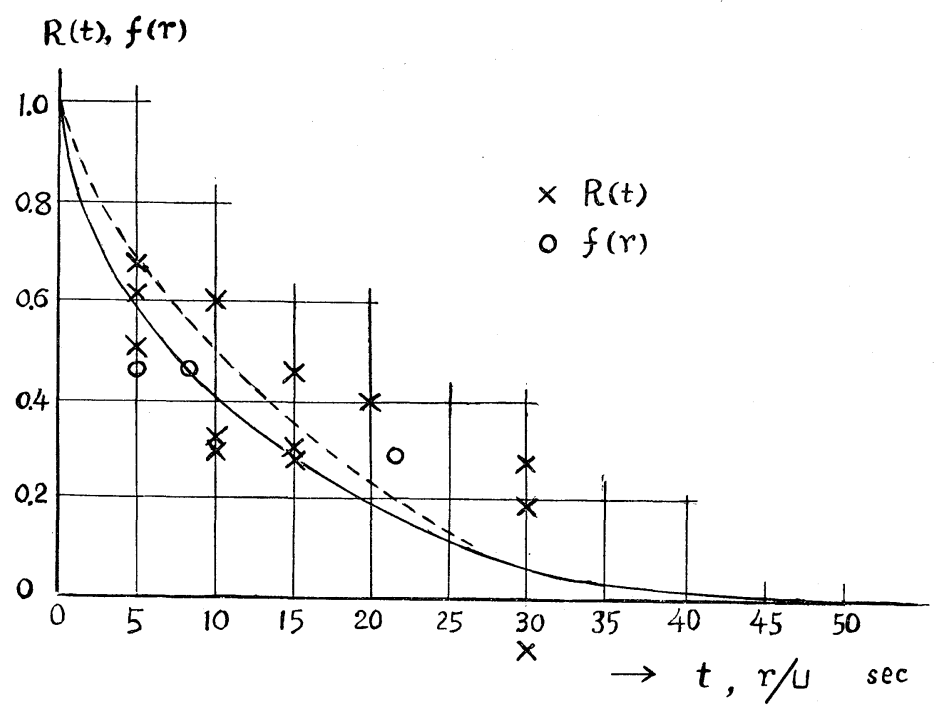

Fig. 3. Eulerian correlation function $R(t)$ and Taylor-Karmanian correlation function $f(r)$. Full line shows our theoretical result $\left(T_{0}, \Lambda_{0}\right.$ are suitably selected). Broken line shows Inoue's $\left(T_{0}, \Lambda_{0}\right.$ are suitably selected) and is reproduced together with his data from Inoue's (1950a). 
where $T_{n}$ is the passage time of turbulons of rank $n$ and $A_{n}$ is the diameter of turbulons of rank $n$; and Inoue's results are corrected as follows :

$$
\begin{aligned}
& \begin{aligned}
R(t) & =1-\left(t / T_{0}\right)^{2 / 3} \quad\left(t<T_{0}\right) \text { into } \\
R(t) & =1-3\left(t / T_{0}\right)^{2 / 3}+2\left(t / T_{0}\right)\left(t<T_{0}\right), \\
f(r) & =1-\left(r / \Lambda_{0}\right)^{2 / 3} \quad\left(r<\Lambda_{0}\right) \text { into } \\
f(r) & =1-3\left(r / \Lambda_{0}\right)^{2 / 3}+2\left(r / \Lambda_{0}\right)\left(r<\Lambda_{0}\right) \\
\text { and } & \\
g(r) & =1-4 / 3\left(r / \Lambda_{0}\right)^{2 / 3} \quad\left(r<\Lambda_{0}\right) \text { into } \\
g(r) & =1-4\left(r / \Lambda_{0}\right)^{2 / 3}+3\left(r / \Lambda_{0}\right)\left(r<\Lambda_{0}\right) .
\end{aligned}
\end{aligned}
$$

The comparison of the results $(R(t)$ and $f(r))$ is shown by Fig. 3 .

\section{Conclusion}

(i) Taking into account the age of turbulons, Inoue's functional form of Lagrangian correlation function

is modified as

$$
R(\xi)=1-\xi / \tau_{0} \quad\left(0<\xi<\tau_{0}\right)
$$

$$
R(\xi)=1-\xi / \tau_{0}+\xi / \tau_{0} \log \left(\xi / \tau_{0}\right) \quad\left(0<\xi<\tau_{0}\right),
$$

which is the same form as that obtained by Grant.

(ii) Allowing an assumption that all the turbulons can be separated effectively into two groups:- that of turbulons contributing only to diffusion of cluster particles and that of turbulons contributing to translation of cluster mass, two-dimensional diffusion of a cluster released instantaneously at a point source is treated and the same results as Batchelor's predicted by similarity principle are obtained.

\section{Acknowledgment}

The writer wishes to express his cordial gratitude to Professor T. Namekawa, the director of the Meteorological Institute, Kyoto University, for his kind advice and encouragement through the course of this work.

\section{References}

Inoue, E. (1950a): On the structure of wind near the ground, J. Met. Soc. Japan, 28, 367-376. (1950b): On the turbulent diffusion in the atmosphere (I). J. Met. Soc. Japan, 28, 441-456.

(1951a): On the turbulent diffusion in the atmosphere (II). J. Met. Soc. Japan, 29, 246-253.

(1951b): On the Lagrangian correlation coefficient for turbulent diffusion and its application to atmospheric diffusion phenomena. Geophy. Res. Pap. 19, 397-413.

(1952): On the structure of wind near the ground. Bull. Nat. Inst. Agri. Sci. Ser. A, No. 2, 1-93.

Batchelor, G. K. (1950): The application of the similarity theory of turbulence to atmospheric diffusion. Q. J. Roy. Met. Soc., 76, 133-146. (1952): Diffusion in a field of homogeneous turbulence. The relative motion of particles. Proc. Camb. Phil. Soc., 48/2, 345-362.

Charnock, H. (1951): Note on eddy diffusion in the atmosphere between one and two kilometers. Q. J. Roy. Met. Soc., 77, 654-658.

Frenkiel, F. N. and Katz, I. (1956): Studies of small-scale turbulent diffusion in the atmosphere. J. Met., 13, 388-394.

Gifford, Jr. F. (1957): Relative atmospheric diffusion of smoke puffs. J. Met., 14, 410-414.

Grant, A.M. (1957): A corrected mixing-length theory of turbulent diffusion. J. Met., 14, 297-303.

Kellogg, W. W. (1956): Diffusion of smoke in the stratosphere. J. Met., 13, 241-250.

Richardson, L.F. (1926): Atmospheric diffusion shown on a distance-neighbour graph. Proc. Roy. Soc. London Ser. A, 110, 709-737.

Taylor, G. I. (1922): Diffusion by continuous movements. Proc. London Math. Soc., 20, 196-212.

\title{
乱流拡散の乱子模型理論の訂正とその応用
}

\author{
松岡春樹 \\ (京都大学気象学特別研究所)
}

井上の乱子模型理論が，乱子の年令を考慮飞入れることとょつて修正され，その結果ラグランジュ相関函数の井上 の形が訂正され，Grant が得たものと一致する。

動源型の相対拡散の乱子理論が，乱子を実効的飞 2 つの群即ち拡散のみ飞寄与する小さい乱子の群と粒子束の中心 の変位にのみ寄与する大きい乱子の群飞分けられるという尤もらしい一仮定の導入により組立てられ，Batchelor が相似仮説によつて予言したのと同じ形の結果が得られた。

Vol. 38, No. 3, 1960 